\title{
Research on Algorithm of Indoor Positioning System Based on Low Energy Bluetooth 4.0
}

\author{
De-Yi ZHANG ${ }^{1}$, Wen-Yue WANG ${ }^{2}$ and Lian-Rong LV $^{1, a}$ \\ ${ }^{1}$ College of Electrical and Electronic Engineering, 300384 Tianjing University of Technology, China \\ ${ }^{2}$ College of computer and Communication Engineering, 300384 Tianjing University of Technology, China
}

\begin{abstract}
This paper mainly analyzes and compares several well-known algorithms for indoor positioning. By many nodes jump tests, relation between RSSI and distance and relation between LQI and packet error rate can be obtained. Then based on the relations, LQI confidence coefficient is classified. By comparing these different kinds of algorithms, this paper perfects the algorithm of fuzzy fingerprint indoor positioning, which can shorten the time required to process off-line data. At last, this algorithm was verified by indoor and outdoor experiments, by which we can know that this algorithm greatly lower RSSI error. And it is concluded that this algorithm has a better positioning accuracy.
\end{abstract}

\section{Introduction}

\subsection{Research background and significance}

Wireless location is an important technology belonging to Things of Internet, which is also a development trend of science and technology in the future. Fuzzy fingerprint positioning algorithm in this paper is based on RSSI (received signal strength indication) and LQI (link quality indicator). which through a certain algorithm to eliminate the signal propagation in the process of loss and error to achieve our desired accuracy, and as far as possible the fingerprint database to reduce the difficulty.

\section{Research of technology concerning indoor positioning system}

\subsection{Several classical indoor positioning technologies}

At present, the mainstream indoor positioning technology is as follows:

(1) Infrared ray positioning: transmission distance is short and the penetrating power is weak; however, it is easily affected by the indoor lighting. Thus, the equipment consumption and the unit price cost will be high.

(2) Ultrasonic wave positioning: being greatly affected by multipath effect, ultrasonic wave will have a positioning error. What's worse, the receiving device will cost a lot.

(3) RFID (radio frequency identification) positioning: propagation distance is short and the devices

\footnotetext{
${ }^{\text {a }}$ Corresponding author: 1631vlianrong@163.com
} 
are difficult to be integrated with general equipment, so it is hard to have a wide popularization.

(4) Ultra-wide band positioning: the equipment costs a lot so it is difficult to be widely used.

(5) BLE(Bluetooth Low Energy) 4.0 positioning: a battery with the same size as a button can provide enough power for the BLE device for one year. The signals from the area which is $100 \mathrm{~m}$ around the BLE can be received. We all know that now almost all cell phones have the newest BLE4.0 module available. Therefore, the mobile terminal with tiny BLE module can be smaller and lighter. In this case, the indoor positioning system based on BLE will have advantages and application values which cannot be available by other indoor positioning technologies.

\subsection{Algorithm for several classical indoor positioning technologies}

\subsubsection{AOA positioning}

The accuracy of AOA positioning will be affected by the sensitivity and 3D resolution of the antenna array. As a result, the places in a city or the indoor places where the arrangement is too crowded, accuracy of AOA positioning will be greatly affected.

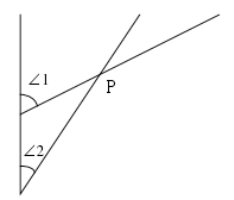

Figure. 1 AOA Positioning Schematic diagram

\subsubsection{TOA positioning}

Time of Arrival positioning is a method to figure out the location of the mobile terminals by three known AP points. TOA greatly depends on the time and the signal transmission has an obvious multipath effect because of the barriers indoor, so TOA is difficult to be widely used for indoor positioning if there is a high requirement on accuracy.

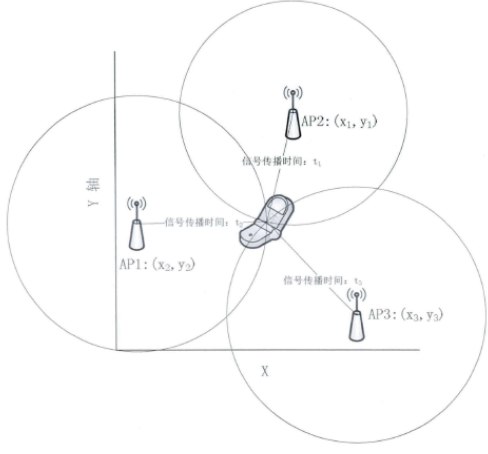

Figure.2 TOA Positioning Schematic Diagram

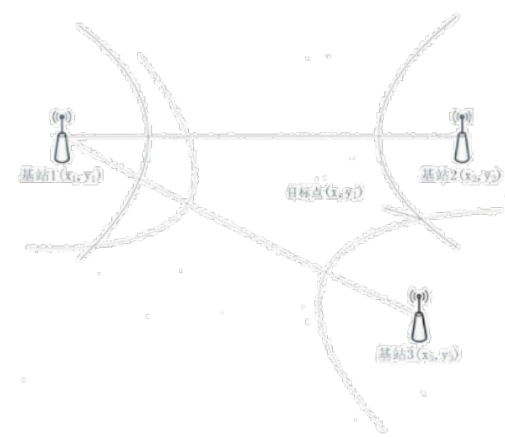

Figure.3 TDOA Positioning Schematic Diagram

\subsubsection{TDOA positioning}

Unlike TOA positioning, TDOA positioning is a method to define the location of mobile terminal by the time gap of signals arriving at the two base stations but not the absolute time of arrival. That is TDOA does not need to have a high accurate clock synchronization between the base station and the 
mobile terminal. In this way, the cost will be lowered. However, it is impossible to fully overcome the adverse affect caused by the complicated indoor environment. That is the signals cannot be transmitted long and the multipath effect is quite obvious, which will seriously affect the accuracy of indoor positioning.

\subsubsection{Fingerprint location positioning}

Compared with other methods, fingerprint positioning is able to fully overcome the problems caused by complicated indoor environment (signals cannot be transmitted far and multipath effect is obvious), which will perfect the allocation of AP points. Because of the complicated indoor environment, multipath transmission of the signals will be much more obvious compared with the signals transmitted at flat outdoor environment. Even though some methods can have a highly accurate positioning outdoor, they cannot be as accurate as fingerprint positioning. The fingerprint location method is just to match the characteristics of multipath propagation, and to a large extent, reduce the error of indoor positioning.

\section{System-based RSSI indoor propagation model}

\subsection{Impact on BLE distribution by distance}

Samples shall be taken from the points which are differently far from the BLE launcher. Each point shall have 100 samples taken. The sampling interval shall be one second. After all the samples are taken, figure out the average value of each BLE sample. See the figure here below for the relationship between RSSI and distance.

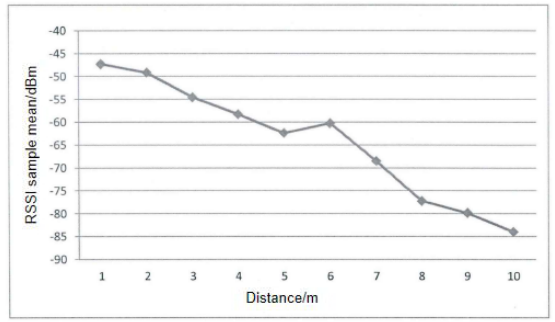

Figure.4 Relationship between RSSI and Distance

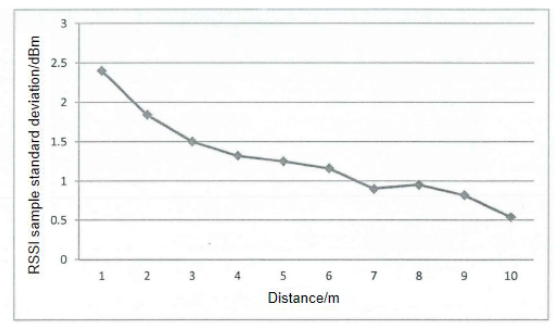

Figure.5 Relationship between Sample Point RSSI and Distance

From Figure 4 (relationship between RSSI and distance), we can see that the signal strength from BLE will be decreased if the distance is increased. However, the decreasing range is getting smaller and smaller. When the signal strength is decreasing, we can see that there is a signal fluctuation. In my opinion, the fluctuation is resulted in by signal diffraction, signal reflection, signal refraction, signal 
scattering or multipath effect. This proves that if signal loss model is used to figure out the distance between sample points indoor, a big error will be caused.

From Figure 5 (Relationship between sample point standard deviation and distance), we can see that as the distance increases, the standard deviation is decreased. That is fluctuation of the samples becomes smaller and smaller when the distance is increasing. Theoretically, the smaller sample fluctuation is, the better fingerprinting positioning will be[2]. This is because that the smaller fluctuation is, the possibility for several sample points to have the same value will be smaller. Thus, combing with Figure 6, I think the places where the standard deviation is small and the average value is higher, it will be much better for fingerprint positioning. Based on my conclusion, here gets the figure showing the relationship between sample point standard deviation and average value. From the figure, we can see that the places which are more than 5m away from BLE will have a more correct positioning. The places which are too close to or too far from the BLE, a correct positioning will be difficult. What's more, we can also see that when the distance is about $5 \mathrm{~m}$, the values shown on RSSI will have a regular curvature. Therefore, we can reduce the time to get data by using Lagrange interpolation polynomial formula. When we use the existing positioning algorithm, the standard deviation of sample signals can be applied. That is we can use different algorithms or fingerprint database to adapt to the demands of different positioning. Therefore, this research selects fuzzy fingerprint for the positioning.

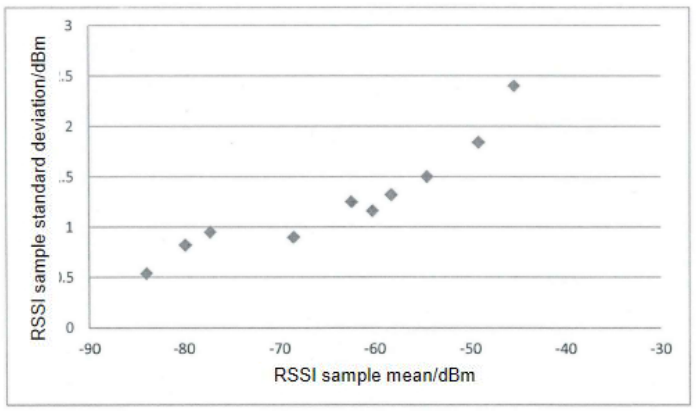

Figure. 6 Relationship between Average Value I and Standard Deviation

\section{Research on BLE 4.0-based fingerprint positioning algorithm}

\subsection{Principle of BLE 4.0-based fingerprint positioning algorithm}

Normally, fingerprint positioning is divided into 2 stages --- offline stage and online real-time positioning stage.

At offline stage, at the designated place whose area is already known, several reference points shall be set. The location of these reference points shall be determined by the environment and the equipment. Then get the signal from these reference points (AP). For example, in this paper, the featured signal that we collect are the BLE RSSI value and LQI value. Combining the signals from these reference points and the Lagrange interpolation polynomial formula, a complete fingerprint offline network for the designated place will be set up. This network is the vector space of these signals. Each signal point will be the location of offline fingerprint existed in the offline fingerprint database. At offline stage, AP points shall be set properly. So if more signals needed to be collected, our workload will be increased[3]. When Matlab simulation, if the algorithm can pass the RSSI value and LQI value, the accuracy of positioning can be as high as $90 \%$; what's more, the algorithm will be much easier (with $1 / \mathrm{c}$ decreased, $\mathrm{c}$ is the coefficient of fuzzy clustering). Here below is the figure showing the procedures of classification: 


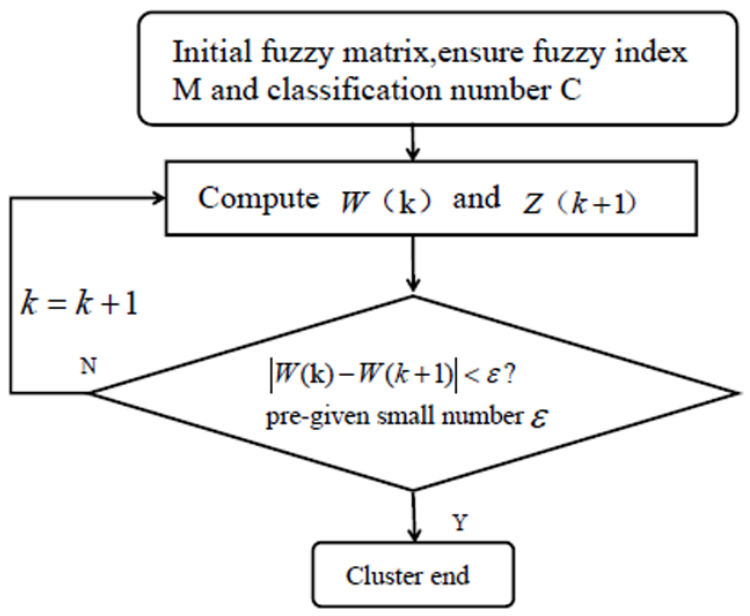

Depending on the iterative process shown above, we can classify the space of data sampled at offline stage: the data space is $\mathrm{C}$, and each data space has $\mathrm{K}$ samples, $\mathrm{k}$ and $\mathrm{z}$ are the center of the fourth space. All the data from space $\mathrm{C}$ form the fingerprint database of offline space.

When the fingerprint database is set up at the offline stage, real-time matching stage shall be started. This stage is to compare and sort out the data and offline fingerprint database. For traditional fingerprint positioning, if the deterministic algorithm is used for positioning, the signals received from designated reference points will be fluctuated (this has been proved aforementioned), so deterministic algorithm cannot well handle this situation. If probabilistic synchronization algorithm, the possibility of signals received at offline stage shall be used as the fingerprint database, and Bayes rule shall be used to judge the location. Since probabilistic synchronization algorithm is based on Bayes rule, which also uses the probability of gaussian distribution, and we can easily proved that the BLE signal strength does not strictly follow gaussian distribution according to the data we got, so it will result in a great error. Therefore, in order to enhance the correctness, fuzzy theory is added into deterministic algorithm.

Online real-time positioning: this is the time when matching the fingerprint. Just like the fuzzy decision, fingerprint matching shall have a modeling via fuzzy decision theory in order to get a more correct positioning. First of all, match the categories and transfer the data at unknown points at the designated places where 4 BLE anchor nodes are measured to the upper computer. Then figure out the fuzzy membership of centers (quantity: C) which have been confirmed by fuzzy clustering. After that, choose the category $\mathrm{j}$ where the maximal fuzzy membership is, do a fuzzy decision. In this case, the location of the unknown point can be defined[3].

\subsection{Simulation verification of positioning algorithm}

At the stage to verify the fuzzy fingerprint algorithm, matlab simulation is used. First of all, the empty place in front of the gate of school of Electronic Engineering, Tianjing University of Technology was selected to collect the offline data. This place is also the one to be positioned by us. Around this empty place,4 BLE 4.0 models were located, which are to be used as the AP points. See the Figure 7 here below for the layout of the 4 models (at the 4 corners of this place). The 4 AP points forms a square with an area of $10 \mathrm{~m}^{*} 10 \mathrm{~m}$. Then, inside the square, points whose interval is $0.5 \mathrm{~m}$ are considered as the supervision. There are 441 points for supervision in total. Then use a BLE 4.0 model with a display to collect the eigenvalue of signals from the 4 AP points, then we can get RSSI and LQI. 


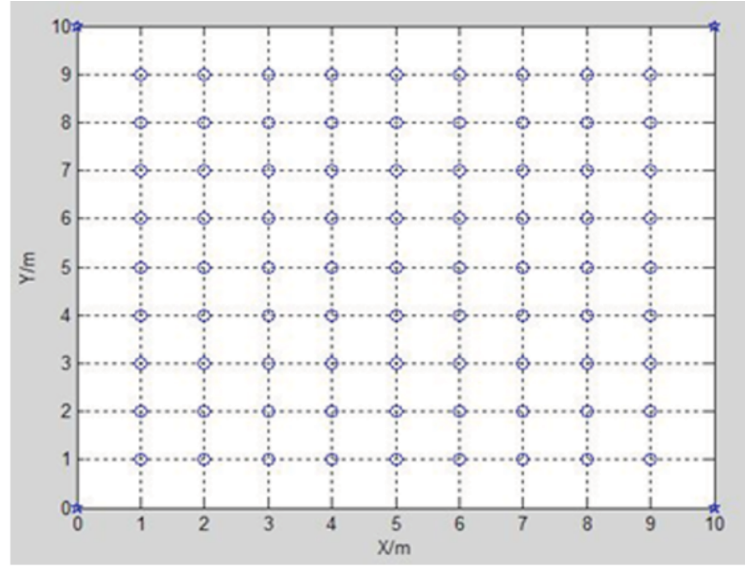

Figure 7. Map of simulation experiment

At the place where we have selected, well set AP points and reference points and then we can start to collect data. We collect 441 groups of data in total (3528 data). They are the RSSI values and LQ values in this paper. Input all the data into Excel so that we can easily read them when matlab simulation. See the figure here below for the save format of part of data.

\begin{tabular}{|c|c|c|c|c|c|c|c|c|c|c|c|}
\hline 4 & A & B & C & D & E & $\mathrm{F}$ & $G$ & H & I & $\mathrm{J}$ & K \\
\hline 2 & 52.6 & 50 & 59.2 & 59.3 & 67.1 & 70.9 & 70.9 & 74.2 & 80.3 & 77.3 & 83.7 \\
\hline 3 & 59.5 & 63.4 & 61.2 & 68.3 & 70 & 71.9 & 77.5 & 78.9 & 77.6 & 78.4 & 81.2 \\
\hline 4 & 62.4 & 63.7 & 71.8 & 68 & 76.7 & 68.9 & 77 & 80.2 & 81 & 80.3 & 77.5 \\
\hline 6 & 72.9 & 76.7 & 78.8 & 71.7 & 75.6 & 73.5 & 78.1 & 81.7 & 78.3 & 81 & 80.6 \\
\hline 7 & 71.5 & 76.4 & 78.9 & 79.2 & 76.5 & 74.5 & 77.5 & 82.7 & 79.4 & 85 & 81.4 \\
\hline 8 & 74.8 & 81 & 81.5 & 76.5 & 81.7 & 75.2 & 78.4 & 81 & 81.3 & 83.1 & 80.8 \\
\hline 9 & 78.3 & 82.5 & 77.6 & 79.3 & 82.8 & 79.4 & 79.4 & 81.8 & 79.3 & 84.8 & 86.4 \\
\hline 12 & 80.4 & 80.6 & 79.3 & 78.3 & 82.4 & 85.4 & 79.9 & 85 & 85.1 & 84.4 & 87.5 \\
\hline 13 & 79.5 & 84.4 & 87 & 86.3 & 83.1 & 81.2 & 84.8 & 85.3 & 84.6 & 85.1 & 84.1 \\
\hline 14 & 83 & 80.2 & 83.1 & 86.8 & 82 & 82.3 & 85.1 & 86.1 & 87.4 & 84.9 & 88 \\
\hline 15 & 83.6 & 83.4 & 87.3 & 85.3 & 87.5 & 83.6 & 88.3 & 87.6 & 88.7 & 85.1 & 86.3 \\
\hline 16 & 84.3 & 82.6 & 86 & 86.6 & 87.3 & 84.3 & 86.4 & 87.5 & 87.7 & 84.9 & 86 \\
\hline 17 & 87.8 & 85.5 & 85.7 & 85.4 & 86.1 & 85.2 & 86.2 & 86.2 & 86.3 & 86.4 & 83.8 \\
\hline 18 & 84.3 & 84.1 & 85.5 & 87.6 & 88.1 & 87.9 & 88.1 & 85.1 & 86.7 & 85.7 & 85.6 \\
\hline
\end{tabular}

From the output result, we can see that at the AP points, the coordinate of a point can be accurately figured out by RSSI value and LQ value if fizzy fingerprint positioning is used.

On the simulated drawing, if the reference points on the actual coordinate $(6.3,2.7)$ are used as the simulation targets, we can get the estimated coordinate $(6.1,2.9)$.

$$
\text { By formula } \Delta=\sqrt{\left(\mathrm{x}^{\prime}-x\right)^{2}+\left(\mathrm{y}^{\prime}-y\right)^{2}}
$$

We can figure out that $\Delta$ is 0.3 . That is the error between the estimated value and the actual value is 0.3 . Therefore, from the data obtained via simulation, the fingerprint positioning algorithm based on fuzzy theory can greatly enhance the positioning accuracy. If we can make sure that the eigenvalue of the signals from mobile terminal will not fluctuate a lot, the gap between the estimated coordinate and the actual coordinate can ensure that the positioning system based on BLE4.0 is able to be widely used for our daily life. At offline stage, the Lagrange interpolation polynomial formula is added, which reduce the collection halfway. Therefore, the calculation workload is greatly lowered. In this way, only one second is needed to figure out the coordinate of the unknown point ( 3 seconds is required by other algorithms). 


\section{Conclusion}

This paper compares and analyzes several well-known indoor positioning algorithms, by which it introduces the advantages and disadvantages of indoor positioning algorithm based on BLE 4.0. After a comprehensive analysis on the requirements of indoor positioning and signal propagation loss, BLE4.0 model is selected as the hardware to establish indoor positioning system. This paper studies several algorithms (AOA, TDOA) which are maturely used by indoor positioning system, and fingerprint positioning algorithm is finally selected as the foundation of our research. After discussing the advantages and disadvantages of fingerprint positioning algorithm, and combining the features of signal transmission, this paper perfects the fuzzy fingerprint positioning algorithm based on the fingerprint positioning algorithm. Fuzzy fingerprint positioning algorithm depends on fuzzy theory. From the results got by simulation, we can know that it greatly simply the calculation and enhance the accuracy and error tolerance of the positioning.

\section{References}

1. ZHANGHao .ZHA0Qian.chuan., JournalofComputerApplicatlons, 31. (2011)

2. Caozhe, Research and implementation of indoor positioning system based on Bluetooth and location fingerprint (2014)

3. LI Juan-juan, Journal of Shanghai University(Natural Science). Fuzzy Fingerprint Location for Bluetooth Specification Version 4.0, 19 (2013)

4. R.W.Jeffre y Hightower, and Gaetano Borrielo, UW Cse,SpotON: An Indoor 3D Location Sensing Technology Based on RD Signal Strengt h.(2000)

5. R.Want, A Hopper,J Gibbons, veronica Falcao and Jonathan Gibbons, Acm, The Active Badge Location Syste m.(2000)

6. Specification of the Bluetooth Core System, www.bluetooth.org

7. Kegen Yu, Ground wireless location technology, 2012

8. Robin Heydon, Bluetooth Low Energy The Developer's Handbook, 2014 